\title{
On confluently graph-like compacta
}

\author{
by \\ Lex G. Oversteegen (Birmingham, AL) and \\ Janusz R. Prajs (Opole and Pocatello, ID)
}

\begin{abstract}
For any class $\mathcal{K}$ of compacta and any compactum $X$ we say that: (a) $X$ is confluently $\mathcal{K}$-representable if $X$ is homeomorphic to the inverse limit of an inverse sequence of members of $\mathcal{K}$ with confluent bonding mappings, and (b) $X$ is confluently $\mathcal{K}$-like provided that $X$ admits, for every $\varepsilon>0$, a confluent $\varepsilon$-mapping onto a member of $\mathcal{K}$. The symbol $\mathbb{L} \mathbb{C}$ stands for the class of all locally connected compacta. It is proved in this paper that for each compactum $X$ and each family $\mathcal{K}$ of graphs, $X$ is confluently $\mathcal{K}$ representable if and only if $X$ is confluently $\mathcal{K}$-like. We also show that for any compactum the properties of: (1) being confluently graph-representable, and (2) being 1-dimensional and confluently $\mathbb{L} \mathbb{C}$-like, are equivalent. Consequently, all locally connected curves are confluently graph-representable. We also conclude that all confluently arc-like continua are homeomorphic to inverse limits of arcs with open bonding mappings, and all confluently tree-like continua are absolute retracts for hereditarily unicoherent continua.
\end{abstract}

1. Introduction. Confluent mappings for compacta, defined by J. J. Charatonik in [2] (very similar classes of mappings were earlier studied in [20]), turned out to be very important. They form a much narrower family than the one of all continuous mappings. However, this family is essentially larger than any of the following classes of mappings: covering, branchcovering, open, or monotone. Yet confluent mappings share some important properties with those listed above. They preserve end points, atriodicity, tree-likeness [13], the property of Kelley and many other topological properties of compacta. They even have (approximate) path lifting properties [7]. The class of confluent mappings is, in a sense, more "regular" than some other well known classes of mappings. For instance, while the inverse limit of open mappings is not necessarily open, the inverse limit (or even the weakly

2000 Mathematics Subject Classification: Primary 54C10, 54C15, 54D05; Secondary $54 \mathrm{~F} 15,54 \mathrm{C} 20$.

Key words and phrases: confluent mappings, locally connected continua, confluently graph-like continua, confluent retraction.

The first author was supported in part by NSF-DMS-0072626. 
induced limit in the sense of Mioduszewski [15]) of confluent mappings is always confluent (cf. $[9,17,10]$ ).

The main purpose of this paper is to study confluently graph-like compacta and to generalize known results for continuous functions to the class of confluent mappings. A compactum $X$ is said to be confluently graph-like provided that for every $\varepsilon>0$ there exists a confluent $\varepsilon$-mapping from $X$ onto a graph. There are many important examples of confluently graph-like compacta. The Menger curve and the Sierpiński universal plane curve are such spaces. More generally, in this paper we prove that all locally connected, 1-dimensional compacta are confluently graph-like (see Corollary 3.16). The solenoids are confluently graph-like (precisely, they form the class of all confluently circle-like continua, see [4]). Inverse limits of arcs with open bonding mappings (called "Knaster type continua") are, obviously, confluently graph-like. Actually, this last class is the class of all confluently arc-like continua (see Corollary 3.4). "Case continua" [1] and their generalizations [18], [14] are also confluently graph-like. In a recent paper [5] it was proved that every inverse limit of trees with confluent bonding mappings is an absolute retract for hereditarily unicoherent continua, and thus it has many other strong properties including the fixed point property (see [7], [3], [6] and [5]). In this paper we show that every confluently tree-like continuum is such a retract (see Corollary 3.7).

Confluently graph-like compacta share many properties with the members of a larger class of all confluently $\mathbb{L} \mathbb{C}$-like compacta, i.e., compacta admitting, for every $\varepsilon>0$, a confluent $\varepsilon$-mapping onto a locally connected compactum ( $\mathbb{L} \mathbb{C}$ stands for the class of all locally connected compacta). For instance such compacta have the arc property of Kelley [4] (cf. Remark 1.1). This last property, satisfied by all locally connected compacta and all absolute retracts for hereditarily unicoherent continua, is interesting in its own right. Confluent mappings on such compacta are known to have some approximate lifting properties [7] (cf. Remark 1.1).

REMARK 1.1. Actually, the main results of papers [4] and [7] are formulated for continua, i.e. connected compacta. However, those mentioned above remain true also for non-connected compacta. The proofs are the same.

Let $\mathcal{K}$ be a class of compacta and $X$ be a compactum. We say that $X$ is confluently $\mathcal{K}$-like provided that $X$ admits, for every $\varepsilon>0$, a confluent $\varepsilon$-mapping onto a member of $\mathcal{K}$. If $X$ is homeomorphic to the inverse limit of an inverse sequence of members of $\mathcal{K}$ with surjective confluent bonding mappings, then $X$ is called a confluently $\mathcal{K}$-representable compactum.

Let $\mathcal{K}$ be a class of compacta. It is an easy observation that if a compactum $X$ is confluently $\mathcal{K}$-representable, then $X$ is confluently $\mathcal{K}$-like. In 
this paper we ask for what classes $\mathcal{K}$ the converse is true. The following question is crucial to this paper.

QUESTION 1.1. Let $\mathcal{K}$ be any of the classes of: polyhedra, ANR's, locally connected compacta, and suppose $X$ is a confluently $\mathcal{K}$-like compactum. Does it follow that $X$ is confluently $\mathcal{K}$-representable?

In what follows we answer this question in the affirmative for each 1dimensional compactum $X$, and this is the main result of the paper. Within the class of continuous functions a similar result was obtained by Mardešić and Segal [12] in 1963. Using this result we obtain a number of conclusions, some of them mentioned above.

Answering Question 1.1 for compacta of dimension greater than 1 is the most important problem of this paper that remains open.

All results described above are presented in Section 3. Section 2 contains some results concerning confluent mappings from locally connected continua onto an arc. Namely, we prove a "confluent" Urysohn's lemma (Theorem 2.12) and confluent extension and retraction theorems (Theorem 2.14 and Corollary 2.15).

Spaces are assumed to be metric and mappings to be continuous in this paper. A (compact) polyhedron of dimension at most 1 is called a graph. If $f: X \rightarrow Y$ is a mapping, $\varepsilon$ is a positive number and $\operatorname{diam}\left(f^{-1}(y)\right)<\varepsilon$ for each $y \in Y$, then $f$ is called an $\varepsilon$-mapping. A mapping $f: X \rightarrow Y$ is said to be confluent provided that for every subcontinuum $K$ of $Y$ and every component $L$ of $f^{-1}(K)$ we have $f(L)=K$. All confluent mappings considered in this paper are surjective and their domain and range spaces are compact.

A continuum $X$ is called arc-like (circle-like tree-like, graph-like) if, for every $\varepsilon>0$, there exists an $\varepsilon$-mapping $f_{\varepsilon}: X \rightarrow Y_{\varepsilon}$, where $Y_{\varepsilon}$ is an arc (a circle, a tree, a graph, respectively). If, additionally, the mappings $f_{\varepsilon}$ are confluent, then we say that $X$ is confluently arc-like (confluently circle-like, confluently tree-like, confluently graph-like, respectively). If $X$ is homeomorphic to the inverse $\operatorname{limit} \lim \left(Y_{n}, f_{n}^{n+1}\right)$, where the mappings $f_{n}^{n+1}: Y_{n+1} \rightarrow Y_{n}$ are confluent and surjective, and the spaces $Y_{n}$ are arcs (circles, trees, graph), then $X$ is called confluently arc-representable (confluently circle-representable, confluently tree-representable, confluently graph-representable, respectively).

A continuum $X$ is said to be hereditarily unicoherent provided that the intersection of any two subcontinua of $X$ is connected. If a hereditarily unicoherent continuum $X$, whenever embedded into another hereditarily unicoherent continuum $Y$, is a retract of $Y$, then we say that $X$ is an absolute retract for hereditarily unicoherent continua. 
A compactum $X$ is said to have the arc property of Kelley provided that for every continuum $K \subset X$, every $p \in K$ and every sequence $p_{n}$ converging to $p$ in $X$ there exists a sequence of arcwise connected continua $K_{n}$ such that $p_{n} \in K_{n}$ and $K_{n}$ converges to $K$ in the sense of the Hausdorff distance.

\section{2. "Confluent" Urysohn's lemma, confluent extensions and re-} tractions for locally connected continua. In this section we study confluent mappings of locally connected continua onto the unit interval $[0,1]$. We will show a stronger, "confluent" variant of the Urysohn lemma for locally connected continua (see Theorem 2.12). This theorem will be applied in the next section. Using similar technique we also obtain extension and retraction theorems for confluent mappings (Theorem 2.14, Corollary 2.15). We add these last two results for completeness of this study. Some results of a similar type concerning classes of mappings related to confluent ones can be found in [21].

To obtain these results we need some preparation. Let $X$ be a continuum. For any surjective mapping $g: X \rightarrow[0,1]$ and any $a \in(0,1)$ let $F(g, a)$ be the union of all components $K$ of $X \backslash g^{-1}(a)$ such that

$$
\text { either } 0<\inf (g(K))<a \text {, or } a<\sup (g(K))<1 .
$$

Lemma 2.1. For any surjective mapping $g: X \rightarrow[0,1]$, where $X$ is a continuum, the following conditions are equivalent:

(1) The mapping $g$ is confluent.

(2) For any $a \in(0,1)$, any component $C$ of $g^{-1}(a)$ and any $\varepsilon>0$ there exists a continuum $L \subset N_{\varepsilon}(C)$ such that $C \subset L$ and $a \in \operatorname{Int}(g(L))$.

(3) The set $F(g, a)$ is empty for each $a \in(0,1)$.

Proof. Let $g=f \circ m$ be the canonical representation of $g$ as the composition of a monotone mapping $m$ and a light mapping $f$. Assume that $g$ is confluent; then it easy to see that $f$ is also confluent. By Lelek and Read [11], $f$ is open. We next show that condition (2) is equivalent to the openness of $f$. Suppose $f$ is open and that $a \in(0,1)$ and $C$ is a component is of $g^{-1}(a)$. Let $J_{n}=[a-1 / n, a+1 / n] \subset(0,1)$ and let $H_{n}$ be the component of $f^{-1}\left(J_{n}\right)$ containing $m(C)$. Then $f\left(H_{n}\right)=J_{n}$. Let $L_{n}=m^{-1}\left(H_{n}\right)$; then $\bigcap L_{n}=C$ and $g\left(L_{n}\right)=J_{n}$. Hence, for $n$ sufficiently large, $L_{n} \subset N_{\varepsilon}(C)$ and (2) holds. Conversely, if (2) holds, then for each $z \in m(X)$ and each open set $U \subset m(X)$ containing $z, f(z) \in \operatorname{Int}(f(U))$ and $f$ is open. Thus (1) is equivalent to (2).

Suppose (3) does not hold, i.e. there exists an $a \in(0,1)$ with $F(g, a) \neq \emptyset$. Thus there is a component $K$ of $X \backslash g^{-1}(a)$ such that either $0<\inf (g(K))<a$, or $a<\sup (g(K))<1$. Suppose $0<\inf (g(K))<a$ (the other case is similar). Let $b \in K$ be such that $c=g(b)=\inf (g(K))$. Let $C$ be the component of 
$g^{-1}(c)$ that contains $b$. Then for any continuum $L$ that contains $C$ and is sufficiently close to $C$ in the sense of the Hausdorff distance, $g(L) \subset K$. Thus $g(L) \subset[c, 1]$ and $c \notin \operatorname{Int}(g(L))$ for such $L$. Thus (2) does not hold. Hence (2) implies (3).

Suppose (2) does not hold, i.e. there are $a \in(0,1)$, a component $C$ of $g^{-1}(a)$ and an $\varepsilon>0$ such that for every continuum $L \subset N_{\varepsilon}(C)$ with $C \subset L$ we have $a \notin \operatorname{Int}(g(L))$. Since $g(L)$ for $L \neq C$ is an interval, $a$ is either the left hand end point or right hand end point of $g(L)$ for all such $L$. Assume the former case (the latter one is similar). Then for sufficiently small $\delta>0$, if we let $a^{\prime}=a+\delta$ then the set $g^{-1}\left(a^{\prime}\right)$ separates $X$ between $C$ and any point $p \in X$ with $g(p)<a$. Hence $C$ is a subset of a component $K$ of $X \backslash g^{-1}\left(a^{\prime}\right)$ such that $0<\min g(K)<a^{\prime}$ and thus $F\left(g, a^{\prime}\right) \neq \emptyset$. So (3) does not hold. This completes the proof of the implication from (3) to (2) and of the entire lemma.

Suppose that $g: X \rightarrow[0,1]$ is a surjective mapping and $X$ is a locally connected continuum. For any $a \in(0,1)$ and any $x \in F(g, a)$ we let $g^{(a)}(x)=a$ and, for $x \in X \backslash F(g, a)$, let $g^{(a)}(x)=g(x)$. By the local connectedness of $X$ the set $N_{\varepsilon}\left(g^{-1}(a)\right)$ contains, for every $\varepsilon>0$, almost all components of $X \backslash g^{-1}(a)$. In particular, this set contains almost all components $K$ of $F(g, a)$. This observation leads to the conclusion that $g^{(a)}: X \rightarrow[0,1]$ is a continuous mapping. Observe that $F(g, a)=\left\{x \in X: g^{(a)}(x) \neq g(x)\right\}$. Define $I(g)=\left\{a \in(0,1): g^{(a)}=g\right\}=\{a \in(0,1): F(g, a)=\emptyset\}$.

In Propositions 2.2-2.11 we assume that $X$ is a locally connected continuum and $g: X \rightarrow[0,1]$ is a surjective mapping. By Lemma 2.1 we observe that such a mapping $g$ is confluent if and only if $I(g)=(0,1)$.

Proposition 2.2. If $g$ is not confluent, then there exists an $\varepsilon>0$ and an open interval $(p, q) \subset[0,1]$ such that for every surjective mapping $h$ : $X \rightarrow[0,1]$ with $d_{\text {sup }}(h, g)<\varepsilon$ we have $(p, q) \cap I(h)=\emptyset$.

Proof. Since $g$ is not confluent, there exists a number $a \in(0,1)$ such that $g^{(a)} \neq g$, or equivalently, $F(g, a) \neq \emptyset$. Let $K$ be a component of $F(g, a)$. Then either $0<\min g(K)<a$, or $a<\max g(K)<1$. Assume $0<\min g(K)<a$ (the proof in the other case is similar). Fix a point $x_{0} \in K$ such that $g\left(x_{0}\right)=$ $\min g(K)$ and two numbers $p, q \in(0,1)$ satisfying $g\left(x_{0}\right)<p<q<a$. Put $\varepsilon=\frac{1}{2} \min \left\{\left|g\left(x_{0}\right)-0\right|,\left|p-g\left(x_{0}\right)\right|,|a-q|\right\}$, and let $h: X \rightarrow[0,1]$ be a surjective mapping such that $d_{\text {sup }}(h, g)<\varepsilon$.

Fix a number $r \in(p, q)$. We will complete the proof by showing that $r \notin I(h)$. Indeed, since $h$ is $\varepsilon$-near to $g$ we have $h(x)>0$ for each $x \in K$, $h\left(x_{0}\right)<p$, and $h(x)>q$ for each $x \in g^{-1}(a)$. Therefore the set $h^{-1}(r)$ separates $\mathrm{Cl}(K)$ between $h\left(x_{0}\right)$ and $\mathrm{Bd}(K)$. Thus the component $K_{1}$ of $X \backslash h^{-1}(r)$ that contains $x_{0}$ satisfies $0<\min h\left(K_{1}\right)<r$. Hence $r \notin I(h)$. 
By the definition we obtain the next proposition.

Proposition 2.3. For each $a \in(0,1)$ we have $g^{(a)}\left(g^{-1}(0)\right)=\{0\}$ and $g^{(a)}\left(g^{-1}(1)\right)=\{1\}$.

Proposition 2.4. For each $a \in(0,1)$ we have $\{a\} \cup I(g) \subset I\left(g^{(a)}\right)$.

Proof. Evidently, we have $\left(g^{(a)}\right)^{(a)}=g^{(a)}$. Thus $a \in I\left(g^{(a)}\right)$.

Suppose $b \notin I\left(g^{(a)}\right)$. Thus $b \in(0,1) \backslash\{a\}$. Then there exists a nonempty component $K$ of $F\left(g^{(a)}, b\right)$. For any $x \in X$ if $g^{(a)}(x) \neq g(x)$, then $g^{(a)}(x)=a$. Using this implication we conclude:

(1) Since neither 0 nor 1 belong to $g^{(a)}(K)$, it follows that neither 0 nor 1 belong to $g(K)$.

(2) Since $g^{(a)}(\operatorname{Bd}(K))=\{b\} \neq\{a\}$, we have $g(\operatorname{Bd}(K))=\{b\}$.

(3) Since $g^{(a)}(K)$ contains some numbers different from $b$, it follows that $g(K)$ contains some numbers different from $b$.

From (1)-(3) it follows that $K$ contains a nonempty component $K_{1}$ of $X \backslash g^{-1}(b)$ such that $0 \notin g\left(K_{1}\right)$ and $1 \notin g\left(K_{1}\right)$. Therefore $K_{1} \subset F(g, b) \neq \emptyset$ and thus $b \notin I(g)$. The proposition is proved.

Proposition 2.5. Let $a, p, q$ be numbers such that $0<p<a<q<1$ and $p, q \in I(g)$. Then $g(F(g, a)) \subset[p, q]$ and $g^{(a)}(F(g, a))=\{a\} \subset(p, q)$.

Proof. The second part of the conclusion follows by the definition. To see the first one suppose, on the contrary, that a component $K$ of $F(g, a)$ contains a point $x$ such that either $g(x)<p$ or $g(x)>q$. Assume $g(x)<p$ (the other case is similar). Then the component of $X \backslash g^{-1}(p)$ that contains $x$ is a subset of $F(g, p)$. On the other hand, $p \in g(I)$ and thus $F(g, p)=\emptyset$, a contradiction.

Proposition 2.6. Let $a_{1}, a_{2}, \ldots$ be a sequence of numbers in $(0,1)$ such that $\operatorname{Cl}\left(\left\{a_{1}, a_{2}, \ldots\right\}\right)=[0,1]$. Then the sequence of mappings $f_{n}: X \rightarrow[0,1]$ defined by $f_{0}=g$ and $f_{n+1}=f_{n}^{\left(a_{n+1}\right)}$ uniformly converges to a confluent mapping $f: X \rightarrow[0,1]$.

Proof. Applying Proposition 2.4 inductively we see that for any $m \geq n$ we have $a_{1}, \ldots, a_{n} \in I\left(f_{m}\right)$. Let $x \in X$. If $a_{i}<f_{n}(x)<a_{j}$ for some $i, j \in\{1, \ldots, n\}$, then according to Proposition 2.5 we have $a_{i}<f_{m}(x)<a_{j}$ for all $m>n$. By the density of $\left\{a_{n}\right\}$ in $[0,1]$ the sequence $f_{n}$ must converge uniformly.

Let $f$ be the limit of this sequence. Since $a_{1}, \ldots, a_{n} \in I\left(f_{m}\right)$ for every $m>n$ and the set $\left\{a_{1}, a_{2}, \ldots\right\}$ is dense in $[0,1]$, there are no numbers $p, q$ as in Proposition 2.2 for $g=f$. Hence $f$ is confluent.

In Propositions 2.7-2.11 we fix a sequence $\left\{a_{n}\right\} \subset(0,1)$ such that $\mathrm{Cl}\left(\left\{a_{1}, a_{2}, \ldots\right\}\right)=[0,1]$, and we assume that mappings $f_{n}$ and $f$ are as 
in Proposition 2.6. For any $p \in X$ let $C_{n}(p)$ be the component of $f_{n}^{-1}\left(f_{n}(p)\right)$ that contains $p$. Define $C(p)$ as the component of $f^{-1}(p)$ that contains $p$, and $X_{0}=\left\{x \in X: f(x)=f_{n}(x)\right.$ for each $\left.n \in\{0,1, \ldots\}\right\}$. Observe that $X_{0}$ is closed in $X$. The proof of the following proposition is straightforward. The details are left to the reader.

Proposition 2.7. For every $p \in X$ we have:

(1) $C_{n}(p) \subset C_{n+1}(p)$.

(2) $\bigcup\left\{C_{n}(p): n \in\{0,1, \ldots\}\right\} \subset C(p)$.

(3) If $p \in X \backslash X_{0}$, then $\bigcup\left\{C_{n}(p): n \in\{0,1, \ldots\}\right\} \backslash X_{0}$ is open in $X$ and $\operatorname{Bd}\left(\left(\bigcup\left\{C_{n}(p): n \in\{0,1, \ldots\}\right\}\right) \backslash X_{0}\right) \subset C(p) \cap X_{0}$.

Proposition 2.8. For every $x \in X$ each component $C$ of the set $C(x) \backslash$ $X_{0}$ is open in $X$. In particular, we have $\operatorname{Bd}(C) \subset X_{0} \cap C(x)$ for such $C$, and thus $C(x) \cap X_{0} \neq \emptyset$ for each $x \in X$.

Proof. Let $z \in C(x) \backslash X_{0}=C(z) \backslash X_{0}$. Then

$$
z \in \operatorname{Int}\left(\bigcup\left\{C_{n}(z): n \in\{0,1, \ldots\}\right\} \backslash X_{0}\right) \subset C(z)=C(x)
$$

by Proposition 2.7, parts (3) and (2), and thus $C(x) \backslash X_{0}$ is open. By the local connectedness of $X$ any component $C$ of $C(x) \backslash X_{0}$ is open as well.

Proposition 2.9. Let $t \in[0,1]$ and suppose $f_{0}^{-1}(t)$ is connected. Then $f^{-1}(t)$ is connected.

Proof. Let $p \in f_{0}^{-1}(t)$. If $a_{1} \neq t$, then $C_{0}(p)=C_{1}(p)=f_{0}^{-1}(t)$. If $a_{1}=t$, then $C_{1}(p)$ may properly contain $f_{0}^{-1}(t)$ but it must remain connected. Similarly, we can argue that $f_{0}^{-1}(t) \subset C_{n}(p)$ for each $n$. Thus the set $f^{-1}(t) \cap X_{0}=f_{0}^{-1}(t)$ is connected. According to Proposition 2.8, for each $x \in f^{-1}(t) \backslash X_{0}$ we have $\emptyset \neq C(x) \cap X_{0} \subset f^{-1}(t) \cap X_{0}$, which completes the proof.

To prove the next proposition we will apply the following lemma.

LEMMA 2.10. Let $Y$ be a closed subset of a locally connected compactum $Z$. If $Y$ is the union of two locally connected sets $A$ and $B$ such that $A$ is open in $Z$, then $Y$ is locally connected.

Proof. First, note that $\mathrm{Bd}(A) \subset B$. Let a sequence $\left\{x_{n}\right\} \subset Y$ converge to a point $x_{0} \in Y$. We prove that for almost all $n$ there are connected sets $K_{n} \subset Y$ such that $x_{0}, x_{n} \in K_{n}$ and $\lim \operatorname{diam}\left(K_{n}\right)=0$, which will complete the proof. Since $A$ is open in $Z$, and $Z$ is locally connected, such $K_{n}$ 's exist for $x_{0} \in A$. Suppose $x_{0} \in Y \backslash A=B \backslash A$. Since $Z$ is a locally connected compactum, it is locally arc connected. Thus, for almost all $n$, there are $\operatorname{arcs} A_{n} \subset Z$ such that $x_{0}, x_{n} \in A_{n}$ and $\lim \operatorname{diam}\left(A_{n}\right)=0$. Let $p_{n}$ be the first point in $A_{n}$ (in the ordering from $x_{n}$ to $x_{0}$ ) such that $p_{n} \notin A$, and $A_{n}^{\prime}$ be the arc in $A_{n}$ from $x_{n}$ to $p_{n}$. Then $p_{n} \in \operatorname{Bd}(A) \subset B$. Since $B$ is 
locally connected, there are connected sets $L_{n} \subset B$ such that $p_{n}, x_{0} \in L_{n}$ and $\lim \operatorname{diam}\left(L_{n}\right)=0$. Note that the sets $K_{n}=A_{n}^{\prime} \cup L_{n}$ are as desired.

Proposition 2.11. Let $t \in[0,1]$ and suppose $f_{0}^{-1}(t)$ is locally connected. Then $f^{-1}(t)$ is locally connected.

Proof. In view of Proposition 2.8 the set $A_{0}=X_{0} \cap f^{-1}(t)$ is nonempty. Applying Proposition 2.7(1), we see that $A_{0}$ is the union of some components of $f_{0}^{-1}(t)$, and thus $A_{0}$ is locally connected. Using again Proposition 2.8 we see that the set $f^{-1}(t) \backslash X_{0}=\bigcup\left\{C(x) \backslash X_{0}: x \in f^{-1}(t)\right\}$ is open in $X$. Thus the compactum $f^{-1}(t)$ is the union of a locally connected set $A_{0}$ and an open subset $f^{-1}(t) \backslash X_{0}$ of $X$. Hence $f^{-1}(t)$ is locally connected by Lemma 2.10 .

TheOREM 2.12. Let $X$ be a locally connected continuum. Then for any two disjoint closed subsets $A$ and $B$ of $X$ there exists a confluent map $f$ : $X \rightarrow[0,1]$ such that $f(A)=\{0\}$ and $f(B)=\{1\}$. Moreover, the map $f$ can be chosen so that in addition:

(1) $f^{-1}(0)$ and $f^{-1}(0)$ are locally connected; and

(2) if $A$ and $B$ are connected, then so are $f^{-1}(0)$ and $f^{-1}(1)$.

Proof. Let $f_{0}: X \rightarrow[0,1]$ be a mapping guaranteed by the Urysohn lemma such that $f_{0}^{-1}(0)=A$ and $f_{0}^{-1}(1)=B$. Let $a_{1}, a_{2}, \ldots$ be a sequence of numbers in $(0,1)$ such that $\operatorname{Cl}\left(\left\{a_{1}, a_{2}, \ldots\right\}\right)=[0,1]$. Inductively define $f_{n+1}=f_{n}^{\left(a_{n+1}\right)}$. By Proposition 2.6 the sequence $f_{n}$ uniformly converges to a confluent mapping $f: X \rightarrow[0,1]$. Applying Proposition 2.3 inductively we see that $f_{n}(A)=\{0\}$ and $f_{n}(B)=\{1\}$ for each $n$. Hence $f(A)=\{0\}$ and $f(B)=\{1\}$.

Now we prove part (1). Since $X$ is a locally connected continuum, every point of $X$ has arbitrarily small closed, connected and locally connected neighborhoods. Thus we can slightly enlarge the sets $A$ and $B$ to locally connected, compact, disjoint sets $A^{\prime}$ and $B^{\prime}$, and apply the previous argument for $A^{\prime}$ and $B^{\prime}$ in place of $A$ and $B$, respectively [16, Proposition 8.7]. Then part (1) follows by Proposition 2.11.

Part (2) follows from Proposition 2.9.

REMARK 2.13. The converse of Theorem 2.12 is false. For example, the harmonic fan $F$ is not locally connected while it is possible to construct for each pair of disjoint closed subsets $A$ and $B$ a confluent map $f: F \rightarrow[0,1]$ to an arc such that $f(A)=0$ and $f(B)=1$.

THEOREM 2.14. Let $X$ be a closed subset of a locally connected continuum $Y$ and $f: X \rightarrow[0,1]$ be a confluent mapping. Then there exists a confluent extension $f^{*}: Y \rightarrow[0,1]$ of the mapping $f$. Moreover, if the sets $f^{-1}(0)$ and $f^{-1}(1)$ are (locally) connected, then we can choose $f^{*}$ so that the sets $\left(f^{*}\right)^{-1}(0)$ and $\left(f^{*}\right)^{-1}(1)$ are also (locally) connected. 
Proof. Since $[0,1]$ is an absolute retract there exists some extension $f_{0}$ : $Y \rightarrow[0,1]$ of the mapping $f$. Let $a_{1}, a_{2}, \ldots$ be a sequence of numbers in $(0,1)$ such that $\operatorname{Cl}\left(\left\{a_{1}, a_{2}, \ldots\right\}\right)=[0,1]$. Inductively define $f_{n+1}=f_{n}^{\left(a_{n+1}\right)}$. By Proposition 2.6 the sequence $f_{n}$ uniformly converges to a confluent mapping $f^{*}: Y \rightarrow[0,1]$. To show that $f^{*} \mid X=f$ it suffices to prove that $f_{n} \mid X=f$ for each $n$. Indeed, by the definition of $f_{0}$ we have $f_{0} \mid X=f$. Suppose $f_{n} \mid X=f$ for some $n$ and let $x \in X$. If $f_{n}(x)=a_{n+1}$, then $f_{n+1}(x)=a_{n+1}=$ $f(x)$. Suppose $x$ belongs to a component $K$ of $Y \backslash f_{n}^{-1}\left(a_{n+1}\right)$. Let $C$ be the component of $X$ that contains $x$. Then the mapping $g=f \mid C: C \rightarrow[0,1]$ is confluent. Since $f_{n}$ is an extension of $f$ (and thus of $g$ ), the set $K$ must contain the component $K^{\prime}$ of $C \backslash g^{-1}\left(a_{n+1}\right)$ that contains $x$. The mapping $g$ is confluent, and thus $F\left(g, a_{n+1}\right)=\emptyset$ (see Lemma 2.1). Therefore either $0 \in g\left(K^{\prime}\right)$ or $1 \in g\left(K^{\prime}\right)$. Consequently, either $0 \in f_{n}(K)$ or $1 \in f_{n}(K)$. This implies that $K \cap F\left(f_{n}, a_{n+1}\right)=\emptyset$. So $f_{n+1}\left|K=f_{n}\right| K$. In particular $f_{n+1}(x)=f_{n}(x)=f(x)$. Hence $f_{n+1} \mid X=f$.

We have proved that $f_{n} \mid X=f$ for each $n$, and thus $f^{*} \mid X=f$. To see the last part of the theorem observe that the extension $f_{0}$ of $f$ can, additionally, satisfy the condition $f_{0}^{-1}(0)=f^{-1}(0)$ and $f_{0}^{-1}(1)=f^{-1}(1)$. Then the conclusion follows by Propositions 2.9 and 2.11. The proof is complete.

COROLlary 2.15. Let $a b$ be an arc in a locally connected continuum $X$ with $a, b$ as the end points of $a b$. Then there exists a confluent retraction $r$ : $X \rightarrow a b$ such that the sets $r^{-1}(a)$ and $r^{-1}(b)$ are locally connected continua.

REMARK 2.16. No nondegenerate continuum different from an arc can replace the segment $[0,1]$ in Theorem 2.14 or the arc $a b$ in Corollary 2.15 . To see this it suffices to show that such a continuum must have each pair of different points joined by a free arc. The details are left to the reader.

3. Confluently graph-like compacta. In this section we prove the main results of the paper. First we prove that for any class $\mathcal{K}$ of graphs and any compactum the properties of being confluently $\mathcal{K}$-representable and of being confluently $\mathcal{K}$-like are equivalent (Theorem 3.2). Then we show (Corollary 3.15 ) that for any compactum the following three conditions are equivalent: (1) being confluently graph-representable, (2) being confluently graphlike, and (3) being 1-dimensional and confluently $\mathbb{L} \mathbb{C}$-like. In particular, this theorem implies that each 1-dimensional locally connected compactum is confluently graph-representable (Corollary 3.16).

Theorem 3.1. Let $f: X \rightarrow F$ be a confluent mapping from a compactum $X$ onto a graph $F$. Then for each $\varepsilon>0$ there is a $\delta>0$ such that for every confluent $\delta$-mapping $g: X \rightarrow G$ from $X$ onto a graph $G$, there exists an onto confluent mapping $h: G \rightarrow F$ satisfying $d_{\text {sup }}(h \circ g, f)<\varepsilon$. 
Proof. Let $e_{1}, \ldots, e_{n}$ be mutually different points in $F$ such that all components of $F \backslash\left\{e_{1}, \ldots, e_{n}\right\}$ are open arcs of diameter less than $\varepsilon / 2$ and any two points $e_{i}, e_{j}$ are the two boundary points of at most one, if any, component of $F \backslash\left\{e_{1}, \ldots, e_{n}\right\}$. Note that, in particular, all end points, all ramification points and all isolated points of $F$ are included in $\left\{e_{1}, \ldots, e_{n}\right\}$. For any $i \in\{1, \ldots, n\}$ define

$L_{i}=\left\{e_{i}\right\} \cup \bigcup\left\{L: L\right.$ is a component of $F \backslash\left\{e_{1}, \ldots, e_{n}\right\}$ with $\left.e_{i} \in \operatorname{Bd}(L)\right\}$.

Notice that $\operatorname{diam}\left(L_{i}\right)<\varepsilon$ for each $i$. Let $\sigma>0$ be such that $N_{\sigma}\left(e_{i}\right) \subset L_{i}$ for each $i \in\{1, \ldots, n\}$. Put $E_{i}=f^{-1}\left(e_{i}\right)$ and $\xi=\min \left\{d_{X}\left(E_{i}, E_{j}\right): i \neq j\right\}$. Fix a number $\delta>0$ such that $\delta<\xi / 2$ and, for any pair $x, y \in X$, we have $d_{F}(f(x), f(y))<\sigma$ whenever $d_{X}(x, y)<\delta$. Let $g: X \rightarrow G$ be a confluent $\delta$-mapping onto a graph $G$. Define $D_{i}=g\left(E_{i}\right)$ for $i \in\{1, \ldots, n\}$ and note that the sets $D_{1}, \ldots, D_{n}$ are mutually disjoint by the definition of $\delta$.

Claim 1. For every component $K$ of $G \backslash\left(D_{1} \cup \ldots \cup D_{n}\right)$, if $\operatorname{Bd}(K) \cap D_{i}$ $\neq \emptyset$ then $f\left(g^{-1}(K)\right) \subset L_{i}$.

Indeed, suppose there exists a point $x \in D_{i} \cap \mathrm{Bd}(K)$ and let $M$ be a component of $g^{-1}(K)$. Since $g$ is confluent, $g(M)=K$ and also $d_{X}\left(M, g^{-1}(x)\right)=0$. Moreover, $g^{-1}(x) \cap E_{i} \neq \emptyset$ and $g$ is a $\delta$-mapping. Therefore $d_{X}\left(M, E_{i}\right)<\delta$. Thus $d_{F}\left(f(M), e_{i}\right)<\sigma$ by the choice of $\delta$. Since the set $f(M)$ is a connected subset of $F \backslash\left\{e_{1}, \ldots, e_{n}\right\}$, the last inequality implies, by the choice of $\sigma$, that $f(M) \subset L_{i}$. Therefore $f(M) \subset L_{i}$ for every component $M$ of $g^{-1}(K)$, and thus the claim is proved.

Claim 2. For every component $K$ of $G \backslash\left(D_{1} \cup \ldots \cup D_{n}\right)$ the set $\operatorname{Bd}(K)$ intersects at most two different sets $D_{i}, D_{j}$. Moreover, if $\operatorname{Bd}(K) \cap D_{i} \neq \emptyset \neq$ $\operatorname{Bd}(K) \cap D_{j}$ and $i \neq j$, then $e_{i}, e_{j}$ are the two different boundary points of a component $L$ of $F \backslash\left\{e_{1}, \ldots, e_{n}\right\}$ and $f\left(g^{-1}(K)\right) \subset L$.

Indeed, this is a consequence of Claim 1 and of the fact that any three different sets $L_{i}, L_{j}, L_{k}$ have empty intersection. Since any two different sets $L_{i}, L_{j}$ are either disjoint or have a component $L$ of $F \backslash\left\{e_{1}, \ldots, e_{n}\right\}$ as their intersection, the last part of the claim follows.

We are ready to define the desired mapping $h: G \rightarrow F$. For any $x \in D_{i}$ put $h(x)=e_{i}$. To define $h$ on $G$ let $K$ be a component of $G \backslash\left(D_{1} \cup \ldots \cup D_{n}\right)$.

First, suppose $D_{i}$, for some fixed $i \in\{1, \ldots, n\}$, is such that $\operatorname{Bd}(K) \subset D_{i}$. Then we put $h(x)=e_{i}$ for each $x \in K$. The only other case is that $\operatorname{Bd}(K) \subset$ $D_{i} \cup D_{j}$ and $\operatorname{Bd}(K) \cap D_{i} \neq \emptyset \neq \operatorname{Bd}(K) \cap D_{j}$ for two different, fixed sets $D_{i}, D_{j}$ such there is a component $L$ of $F \backslash\left\{e_{1}, \ldots, e_{n}\right\}$ with $e_{1}, e_{2}$ as their boundary points (see Claim 2). In this case we apply Theorem 2.12 for the graph $\mathrm{Cl}(K)$ with two disjoint nonempty subsets $\mathrm{Bd}(K) \cap D_{i}, \operatorname{Bd}(K) \cap D_{j}$ and obtain a confluent mapping $h$ from $\mathrm{Cl}(K)$ to $L \cup\left\{e_{1}, e_{2}\right\}$ such that $h\left(D_{i}\right)=\left\{e_{i}\right\}$ and $h\left(D_{j}\right)=\left\{e_{j}\right\}$. We have defined $h(x)$ for each $x \in G$. 
Notice that $h: G \rightarrow F$ is well defined, continuous and onto.

Claim 3. $d_{\text {sup }}(h \circ g, f)<\varepsilon$.

Indeed, let $x \in X$. If $x \in g^{-1}\left(D_{i}\right)$ for some $i$, then $h \circ g(x)=e_{i}$ and $d_{X}\left(x, E_{i}\right)<\delta$. Thus $d_{F}\left(f(x), e_{i}\right)<\sigma$ by the choice of $\delta$. Therefore $f(x) \in L_{i}$ by the choice of $\sigma$, and thus $d_{F}\left(f(x), e_{i}\right)=d_{F}(f(x), h \circ g(x))<\varepsilon$.

Now assume that $x \in X \backslash\left(g^{-1}\left(D_{1}\right) \cup \ldots \cup g^{-1}\left(D_{n}\right)\right)$. Then $g(x)$ belongs to a component $K$ of $G \backslash\left(D_{1} \cup \ldots \cup D_{n}\right)$. This component must satisfy $\operatorname{Bd}(K) \cap D_{i} \neq \emptyset$ for some $i$. By the definition of $h$ we have $h(\mathrm{Cl}(K)) \subset \mathrm{Cl}\left(L_{i}\right)$ and $f(x) \in L_{i}$ (compare Claim 1). Since $\operatorname{diam}\left(\mathrm{Cl}\left(L_{i}\right)\right)<\varepsilon$ we again have $d_{F}(f(x), h \circ g(x))<\varepsilon$, which completes the proof of the claim.

It remains to show that $h$ is confluent. Suppose, on the contrary, that there exists a continuum $P$ in $F$ and a component $Q$ of $h^{-1}(P)$ such that $h(Q)$ is a proper subset of $P$. Let $p$ be a boundary point of $h(Q)$ in $P$ and fix a point $q$ in $Q \cap h^{-1}(p)$. Choose an arc $A$ in $P$ such that

(i) $p$ is an end point of $A$;

(ii) $A \cap h(Q)=\{p\}$;

(iii) if $p \in F \backslash\left\{e_{1}, \ldots, e_{n}\right\}$, then $A \subset F \backslash\left\{e_{1}, \ldots, e_{n}\right\}$;

(iv) if $p=e_{i}$ for some $i$, then $A \subset L_{i}$.

Then the component $C$ of $h^{-1}(A)$ that contains $q$ satisfies $h(C)=\{p\}$. Suppose first that $C \cap\left(D_{1} \cup \ldots \cup D_{n}\right)=\emptyset$. Then $C$ is contained in a component $K$ of $G \backslash\left(D_{1} \cup \ldots \cup D_{n}\right)$. Put $K^{\prime}=\mathrm{Cl}(K)$ and let $\left\{a_{n}\right\}$ be a sequence of points in $A$ converging to $p, A_{n}=\left[p, a_{n}\right] \subset A$ and let $B_{n}$ be the component of $h^{-1}\left(A_{n}\right)$ containing $C$. Then $B_{n} \subset Q$ and there exists an $n$ such that $B_{n} \subset K$. Since $h \mid K^{\prime}$ is confluent, $h\left(B_{n}\right)=A_{n}$. This contradicts the definition of $p$ and $A$.

Hence we may assume that there exists an $i$ such that $C \cap D_{i} \neq \emptyset$ and thus $h(C)=e_{i}$. Note that $A$ is contained in the closure $L^{\prime}$ of a unique component $L$ of $F \backslash\left\{e_{1}, \ldots, e_{n}\right\}$ with end points $e_{i}$ and $e_{j}$. Choose $r \in C \cap D_{i}$ and $u \in g^{-1}(r) \cap E_{i}$. Let $B$ be the component of $f^{-1}\left(L^{\prime}\right)$ which contains the point $u$. Since $f$ is confluent, $f(B)=L^{\prime}$, and hence $g(B)$ is a subgraph of $G$ which meets $D_{i}, D_{j}$ and $r \in g(B) \cap C$.

Let $\mathcal{K}_{i, j}$ denote the union of $D_{i}, D_{j}$ and all components $K$ of $G \backslash\left(D_{1} \cup\right.$ $\left.\ldots \cup D_{n}\right)$ such that $\partial K \subset D_{i} \cup D_{j}$.

Claim 4. The set $g(B)$ is contained in $\mathcal{K}_{i, j}$.

Indeed, if a component $K$ of $G \backslash\left(D_{1} \cup \ldots \cup D_{n}\right)$ meets $g(B)$ at a point $x$, then $f\left(g^{-1}(K)\right) \cap L \neq \emptyset$. By Claims 1 and 2 the set $f\left(g^{-1}(K)\right)$ must be contained in some set $L_{k}$. But $L_{k} \cap L \neq \emptyset$ only in the case $k \in\{i, j\}$. Therefore $f\left(g^{-1}(K)\right) \subset L_{i} \cup L_{j}$. We also have $\operatorname{Bd}(K) \cap D_{m}=\emptyset$ for each $m$ 
different from $i$ and $j$ because otherwise $f\left(g^{-1}(K)\right) \subset L_{m}$ (see Claim 1) and $L_{m} \cap L=\emptyset$, which is impossible.

Let $\mathcal{K}_{i}$ denote the union of $D_{i}$ and all components $K$ of $G \backslash\left(D_{1} \cup \ldots \cup D_{n}\right)$ such that $\partial K \subset D_{i}$. Note that $h\left(\mathcal{K}_{i}\right)=e_{i}$ by the definition of $h$. Recall that $r \in C \cap D_{i}$. Let $\mathcal{K}_{i}(r)$ be the component of $\mathcal{K}_{i}$ which contains $r$. Then $\mathcal{K}_{i}(r) \subset Q$. Since $g(B)$ meets $D_{j}$ it follows from Claim 4 that there exists a component $K$ of $G \backslash\left(D_{1} \cup \ldots \cup D_{n}\right)$ such that $z \in \mathrm{Cl}(K) \cap \mathcal{K}_{i}(r) \neq \emptyset \neq$ $\partial K \cap D_{j}$. Hence $h(\mathrm{Cl}(K))=\mathrm{Cl}(L)$ and $h \mid \mathrm{Cl}(K)$ is confluent. Let $D$ be the component of $h^{-1}(A)$ containing the point $z$; then $h(D)=A$, and hence $A \subset h(Q)$. This contradicts the choice of $p$ and $A$, and completes the proof of the theorem.

THEOREM 3.2. Let $\mathcal{K}$ be any class of graphs and $X$ be a compactum. Then $X$ is confluently $\mathcal{K}$-representable if and only if $X$ is confluently $\mathcal{K}$-like.

Proof. Suppose that $\mathcal{K}$ is a class of graphs such that for each $\varepsilon>0$ there exists a confluent $\varepsilon$-map from the compactum $X$ onto a member of $\mathcal{K}$. We may assume by Theorem 3.1 that there exists a sequence $\varepsilon_{n} \rightarrow 0$, a countable family of graphs $G_{n}$, confluent onto mappings $f_{n}: X \rightarrow G_{n}$ and $g_{n}^{n+1}: G_{n+1} \rightarrow G_{n}$ such that

(1) $X \cup \bigcup_{n} G_{n}$ is a subset of the Hilbert cube $Q$ with metric $d$,

(2) $d\left(x, f_{n}(x)\right)<\varepsilon_{n}$ for each $x \in X$ and each $n$,

(3) $d\left(g_{k}^{m}\left(f_{m}(x)\right), g_{k}^{n}\left(f_{n}(x)\right)\right)<e_{k}$ for each $k \leq m \leq n$ and each $x \in X$.

By [15], there exists a continuous and onto mapping $f: X \rightarrow \lim \left(G_{n}, g_{n}^{n+1}\right)$ defined by $f(x)=\left(y_{k}\right)$, where $y_{k}=\lim _{n \rightarrow \infty} g_{k}^{n}\left(f_{n}(x)\right)$ for all $\overleftarrow{k}$. It suffices to show that $f$ is one-to-one. Let $\pi_{n}: \lim \left(X_{n}, g_{n}^{n+1}\right) \rightarrow X_{n}$ denote the natural projection. Suppose that $x, y \in \overleftarrow{X}$ are such that $d(x, y)>\eta>0$. Choose $n$ such that $\sum_{i=n}^{\infty} \varepsilon_{i}<\eta / 6$. Then $d\left(\pi_{n}(f(x)), \pi_{n}(f(y))\right)>d(x, y)-$ $4 \sum_{i=n}^{\infty} \varepsilon_{i}>0$. This completes the proof of the theorem.

Using a different approach the following result about confluently circlelike continua was also proved in [4].

Corollary 3.3. A continuum $X$ is confluently circle-like if and only if $X$ is a solenoid.

The next result answers a question from [4].

COROllary 3.4. A continuum $X$ is confluently arc-like if and only if $X$ is a Knaster type continuum, i.e. $X$ is homeomorphic to an inverse limit of arcs with open bonding mappings.

Let $\mathbb{L} \mathbb{C}$ stand for the class of all locally connected compacta.

Corollary 3.5. An atriodic continuum $X$ is confluently $\mathbb{L} \mathbb{C}$-like if and only if $X$ is either a solenoid or a Knaster type continuum. 
Corollary 3.6. Each confluently tree-like continuum is confluently tree-representable.

In a recent paper [5] it was proved that confluently tree-representable continua are absolute retracts for hereditarily unicoherent continua. The question whether the same is true for confluently tree-like ones was an important inspiration to the research presented here. The next result answers this question in the affirmative.

COROLlaRY 3.7. Each confluently tree-like continuum is an absolute retract for hereditarily unicoherent continua.

REMARK 3.8. It is known that a confluently $\mathbb{L} \mathbb{C}$-like continuum must have the property of Kelley [4]. In particular, a confluently tree-like dendroid $X$ has the property of Kelley (consequently, $X$ must be smooth). In view of Corollary 3.7, the question whether every dendroid with the property of Kelley is confluently tree-like is of some interest (compare [5]). Recently, the second named author and Włodzimierz Charatonik found an example of a non-confluently tree-like dendroid with the property of Kelley. The same example is also a candidate for a non-confluently tree-like absolute retract for tree-like continua. These results will be presented in a future paper.

Let $G$ be a graph and $S$ be a simplicial complex structure on $G$. The 0 -dimensional elements of such a structure will be denoted by $\left\{e_{1}\right\}, \ldots,\left\{\varepsilon_{n}\right\}$ and points $e_{1}, \ldots, e_{n}$ will also be called vertices of $S$. Denote by $\mathcal{S}(G)$ the family of all simplicial complex structures $S$ such that each pair of two different vertices $e_{i}, e_{j}$ of $S$ can be boundary points of at most one, if any, 1-dimensional element of $S$. For any $S \in \mathcal{S}(G)$ and vertex $e_{i}$ of $S$, let

$$
L_{i}(S)=\left\{e_{i}\right\} \cup \bigcup\left\{\operatorname{Int}(F): F \in S, \operatorname{dim} F=1 \text { and } e_{i} \in \operatorname{Bd}(F)\right\},
$$

and, if $F \in S$ with $\operatorname{dim} F=1$,

$L_{F}(S)=L_{i}(S) \cup L_{j}(S), \quad$ where $e_{i}, e_{j}$ are the boundary points of $F$.

If $F=\{v\}=\left\{e_{i}\right\}$, i.e., $v=e_{i}$ is a vertex of $S$, we also write $L_{i}(S)=L_{v}(S)=$ $L_{F}(S)$. Let $f: X \rightarrow G$ be a mapping, where $X$ is compact. We say that a family $\mathcal{P}$ of sets refines the family $\mathcal{Q}$ if for each $P \in \mathcal{P}$ there exists $Q \in \mathcal{Q}$ such that $P \subset Q$. If $\mathcal{Q}$ is a family of sets in $X$ and $f: X \rightarrow Y$ is a mapping such that the family $\left\{f^{-1}(y): y \in Y\right\}$ refines $\mathcal{Q}$, we say that the mapping $f$ refines $\mathcal{Q}$. The following observation follows from the compactness of the domain of the function $f$.

ObSERVATION 3.9. If $\mathcal{U}$ is a family of open sets covering a compactum $X$ and $f: X \rightarrow G$ is a mapping to a graph $G$ such that $f$ refines $\mathcal{U}$, then there exists $S \in \mathcal{S}(G)$ such that the family $\left\{f^{-1}\left(L_{F}(S)\right): F \in S\right\}$ refines $\mathcal{U}$. 
LEMMA 3.10. Let $\mathcal{U}$ be an open cover of a locally connected compactum $X$ and suppose $X$ admits a mapping, that refines $\mathcal{U}$, onto a graph $H$. Then there exists a graph $G$, a structure $S \in \mathcal{S}(G)$ and a surjective mapping $g: X \rightarrow G$ such that the family $\left\{g^{-1}\left(L_{F}(S)\right): F \in S\right\}$ refines $\mathcal{U}$ and the set $g^{-1}(F)$ is connected for each $F \in S$.

Proof. We begin with the following claim.

ClaIM 1. There exists a graph $H$, a mapping $h: X \rightarrow H$ and a structure $S \in \mathcal{S}(H)$ such that the family $\left\{h^{-1}\left(L_{F}(S)\right): F \in S\right\}$ refines $\mathcal{U}$ and $h^{-1}(v)$ has finitely many components only for each vertex $v$ of $S$.

Indeed, let $h_{1}: X \rightarrow H$ be a mapping onto a graph $H$ that refines $\mathcal{U}$. By Observation 3.9 there exists a structure $S \in \mathcal{S}(H)$ such that the family $\left\{h_{1}^{-1}\left(L_{F}(S)\right): F \in S\right\}$ refines $\mathcal{U}$. Let $\xi$ be the minimum of the numbers $d_{X}\left(h_{1}^{-1}\left(e_{i}\right), h_{1}^{-1}\left(X \backslash L_{i}(S)\right)\right)$ for all vertices $e_{i}$ of $S$. Since $X$ is a locally connected compactum and $h_{1}^{-1}\left(\left\{e_{1}, \ldots, e_{n}\right\}\right)$ is compact, there exists a finite collection of continua $K_{1}, \ldots, K_{m}$ in $X$ such that $\operatorname{diam}\left(K_{j}\right)<\xi$, $h_{1}^{-1}\left(\left\{e_{1}, \ldots, e_{n}\right\}\right) \cap K_{j} \neq \emptyset$ for each $j \in\{1, \ldots, m\}$, and $h_{1}^{-1}\left(\left\{e_{1}, \ldots, e_{n}\right\}\right) \subset$ $K_{1} \cup \ldots \cup K_{m}$. The condition $\operatorname{diam}\left(K_{j}\right)<\xi$ implies that for each $j \in$ $\{1, \ldots, m\}$ there exists exactly one $i \in\{1, \ldots, n\}$ such that $K_{j} \cap h_{1}^{-1}\left(e_{i}\right) \neq \emptyset$, and we have $K_{j} \subset h_{1}^{-1}\left(L_{i}(S)\right)$ for such $i$. Define $M_{i}=\bigcup\left\{K_{j}: K_{j} \cap h_{1}^{-1}\left(e_{i}\right) \neq \emptyset\right\}$ for $i \in\{1, \ldots, n\}$. For any $F \in S$ with $\operatorname{dim} F=1$ and with end points $e_{i}, e_{j}$ let $P_{i, j}=M_{i} \cup M_{j} \cup h_{1}^{-1}(F)$ and observe that $P_{i, j}$ is compact. Then by the Urysohn lemma there exists a mapping $h_{i, j}: P_{i, j} \rightarrow F$ such that $h_{i, j}^{-1}\left(e_{i}\right)=M_{i}$ and $h_{i, j}^{-1}\left(e_{j}\right)=M_{j}$. For each $F \in S$ with $\operatorname{dim} F=1$ and $\operatorname{Bd}(F)=\left\{e_{i}, e_{j}\right\}$, and for each $x \in M_{i} \cup M_{j} \cup h_{1}^{-1}(F)$, we define $h(x)=h_{i, j}(x)$. Observe that $h: X \rightarrow H$ is a well defined continuous function. Moreover, the set $h^{-1}\left(e_{i}\right)=M_{i}$ is the finite union of continua, and $h^{-1}\left(L_{F}(S)\right) \subset h_{1}^{-1}\left(L_{F}(S)\right)$ for 1-dimensional sets $F \in S$. Hence the family $\left\{h^{-1}\left(L_{F}(S)\right): F \in S\right\}$ refines $\mathcal{U}$, which completes the proof of the claim.

For any graph $H$, any structure $S \in \mathcal{S}(H)$ and any mapping $h: X \rightarrow H$ such that the family $\left\{h^{-1}\left(L_{F}(S)\right): F \in S\right\}$ refines $\mathcal{U}$ let $k(h, S)$ be the difference between the number of components of $h^{-1}\left(\left\{e_{1}, \ldots, e_{n}\right\}\right)$ and the number of nonempty sets $h^{-1}\left(\left\{e_{i}\right\}\right)$. Fix a graph $G_{1}$, a structure $S_{1} \in \mathcal{S}\left(G_{1}\right)$ with vertices $e_{1}, \ldots, e_{n}$, and a mapping $g_{1}: X \rightarrow G_{1}$ such that the family $\left\{g_{1}^{-1}\left(L_{F}\left(S_{1}\right)\right): F \in S_{1}\right\}$ refines $\mathcal{U}$ and the number $k\left(g_{1}, S_{1}\right)$ is minimal. By Claim $1, k\left(g_{1}, S_{1}\right)$ is finite.

Claim 2. For every $i \in\{1, \ldots, n\}$ any two different components of $g_{1}^{-1}\left(e_{i}\right)$ are subsets of two different components of $g_{1}^{-1}\left(L_{i}\left(S_{1}\right)\right)$.

Suppose, on the contrary, that two different components of $g_{1}^{-1}\left(e_{i}\right)$ are contained in the same component of $g_{1}^{-1}\left(L_{i}\left(S_{1}\right)\right)$. Since components of open subsets of locally connected compacta are arcwise connected and $g_{1}^{-1}\left(L_{i}\left(S_{1}\right)\right)$ 
is open in $X$, there exists an arc $a b$ in $g_{1}^{-1}\left(L_{i}\left(S_{1}\right)\right)$ joining two different components $A$ and $B$ of $g_{1}^{-1}\left(e_{i}\right)$ such that $a b \cap g_{1}^{-1}\left(e_{i}\right)=\{a, b\}, a \in A$, and $b \in B$. Let $K_{1}, \ldots, K_{m}$ be all components of $g_{1}^{-1}\left(\left\{e_{1}, \ldots, e_{n}\right\}\right)$ with $K_{m-1}=A$ and $K_{m}=B$. Define $K_{m-1}^{\prime}=A \cup a b \cup B$. Using the continua $K_{1}, \ldots, K_{m-2}, K_{m-1}^{\prime}$ we modify the mapping $g_{1}$ to a mapping $g_{1}^{\prime}: X \rightarrow G_{1}$ (in the same way as the mapping $h_{1}$ was modified to the mapping $h$ in the proof of Claim 1) so that the family $\left\{\left(g_{1}^{\prime}\right)^{-1}\left(L_{F}\left(S_{1}\right)\right): F \in S_{1}\right\}$ refines $\mathcal{U}$ and $K_{1}, \ldots, K_{m-2}, K_{m-1}^{\prime}$ are all components of $\left(g_{1}^{\prime}\right)^{-1}\left(\left\{e_{1}, \ldots, e_{n}\right\}\right)$. Thus we have $k\left(g_{1}^{\prime}, S_{1}\right)<k\left(g_{1}, S_{1}\right)$. So $k\left(g_{1}, S_{1}\right)$ is not minimal, a contradiction.

Now we will prove that $k\left(g_{1}, S_{1}\right)=0$. Suppose, on the contrary, that $k\left(g_{1}, S_{1}\right)>0$. Thus the set $g_{1}^{-1}\left(e_{i}\right)$ is not connected for some $i$. Without loss of generality we can assume that $g_{1}^{-1}\left(e_{n}\right)$ is not connected. Let $D$ be a component of $g_{1}^{-1}\left(e_{n}\right)$ and $W$ be the component of $g_{1}^{-1}\left(L_{n}\left(S_{1}\right)\right)$ that contains $D$. Using Claim 2 we see that other components of $g_{1}^{-1}\left(e_{n}\right)$ are contained in components of $g_{1}^{-1}\left(L_{n}\left(S_{1}\right)\right)$ different from $W$.

Let $G_{1}^{\prime}$ be a homeomorphic copy of $G_{1}$ disjoint from $G_{1}$, and $f: G_{1} \rightarrow G_{1}^{\prime}$ be a homeomorphism. For each $x \in X \backslash W$ we identify the pair $x$ and $f(x)$ in the disjoint union $G_{1} \cup G_{1}^{\prime}$. Let $q: G_{1} \cup G_{1}^{\prime} \rightarrow q\left(G_{1} \cup G_{1}^{\prime}\right)$ be the quotient map of this identification. Put $G_{2}=q\left(G_{1} \cup G_{1}^{\prime}\right)$ and $e_{n+1}=(f \circ q)\left(e_{n}\right)$. We will identify the graph $G_{1}$ with $q\left(G_{1}\right)$ by the homeomorphism $q \mid G_{1}$. So $G_{1} \subset G_{2}$. Let $S_{2} \in \mathcal{S}\left(G_{2}\right)$ be the simplicial complex structure on $G_{2}$ introduced by vertices $e_{1}, \ldots, e_{n}, e_{n+1}$. Define $g_{2}(x)=g_{1}(x)$ for $x \in X \backslash W$, and $g_{2}(x)=\left(g_{1} \circ q \circ f\right)(x)$ for $x \in W$. Observe that $g_{2}: X \rightarrow G_{2}$ is a well defined, continuous mapping. Moreover, $g_{2}^{-1}\left(L_{i}\left(S_{2}\right)\right) \subset g_{1}^{-1}\left(L_{i}\left(S_{1}\right)\right)$ for $i \in$ $\{1, \ldots, n\}$ and $g_{2}^{-1}\left(L_{n+1}\left(S_{2}\right)\right) \subset g_{1}^{-1}\left(L_{n}\left(S_{1}\right)\right)$. This implies that the family $\left\{g_{2}^{-1}\left(L_{F}\left(S_{2}\right)\right): F \in S_{2}\right\}$ refines $\mathcal{U}$. Note that the sets $g_{1}^{-1}\left(\left\{e_{1}, \ldots, e_{n}\right\}\right)$ and $g_{2}^{-1}\left(\left\{e_{1}, \ldots, e_{n}, e_{n+1}\right\}\right)$ are identical while the number of nonempty sets $g_{2}^{-1}\left(e_{i}\right)$ (for $i \in\left\{e_{1}, \ldots, e_{n}, e_{n+1}\right\}$ ) is 1 greater than the number of nonempty sets $g_{1}^{-1}\left(e_{i}\right)$ (for $\left.i \in\{1, \ldots, n\}\right)$. So $k\left(g_{2}, S_{2}\right)<k\left(g_{1}, S_{1}\right)$, and thus $k\left(g_{1}, S_{1}\right)$ is not minimal, a contradiction.

We have proved that $k\left(g_{1}, S_{1}\right)=0$. Thus $g_{1}^{-1}\left(e_{i}\right)$ is connected for each $i \in\{1, \ldots, n\}$. Now we are ready to define the required mapping $g$. Let $x \in g_{1}^{-1}\left(\left\{e_{1}, \ldots, e_{n}\right\}\right)$. Then we put $g(x)=g_{1}(x)$. Let $P$ be a component of $G_{1} \backslash\left\{e_{1}, \ldots, e_{n}\right\}$ with the boundary points $e_{i}, e_{j}$, and let $x$ belong to a component $Q$ of $g_{1}^{-1}(P)$. If $\operatorname{Bd}(Q) \cap g_{1}^{-1}\left(e_{i}\right) \neq \emptyset \neq \operatorname{Bd}(Q) \cap g_{1}^{-1}\left(e_{j}\right)$, we put $g(x)=g_{1}(x)$. If $\operatorname{Bd}(Q)$ meets $g_{1}^{-1}\left(e_{i}\right)$ only, we put $g(x)=e_{i}$, and if $\operatorname{Bd}(Q)$ meets $g_{1}^{-1}\left(e_{j}\right)$ only, we put $g(x)=e_{j}$. Since $X$ is locally connected, $g$ is a well defined continuous mapping into $G_{1}$. Define $G=g(X)$ and let $S \in \mathcal{S}(G)$ be the simplicity complex structure on $G$ introduced by the set of vertices $E=$ $\left\{e_{1}, \ldots, e_{n}\right\} \cap G$. Then for each $e_{i} \in E$ we have $g^{-1}\left(L_{i}(S)\right) \subset g_{1}^{-1}\left(L_{i}\left(S_{1}\right)\right)$, 
and thus the family $\left\{g^{-1}\left(L_{F}(S)\right): F \in S\right\}$ refines $\mathcal{U}$. By the construction the set $g^{-1}\left(e_{i}\right)$ is connected for every $e_{i} \in E$. If $F \in S, \operatorname{dim} F=1$ and $e_{i}, e_{j}$ are the boundary points of $F$, then every component $Q$ of $g^{-1}\left(F \backslash\left\{e_{i}, e_{j}\right\}\right)$ satisfies $g(Q)=F \backslash\left\{e_{i}, e_{j}\right\}$. Employing this last observation and the fact that the sets $g^{-1}\left(e_{i}\right), g^{-1}\left(e_{j}\right)$ are connected, we conclude that $g^{-1}(F)$ is also connected. The proof is complete.

THEOREM 3.11. Let $\mathcal{U}$ be an open cover of a locally connected continuum $X$ and suppose $X$ admits a mapping that refines $\mathcal{U}$ onto a graph. Then there exists a graph $G$, a structure $S \in \mathcal{S}(G)$ and a confluent mapping $g: X \rightarrow G$ such that the family $\left\{g^{-1}\left(L_{F}(S)\right): F \in S\right\}$ refines $\mathcal{U}$ and the set $g^{-1}(F)$ is a locally connected, connected continuum for each $F \in S$.

Proof. First, let $g^{\prime}: X \rightarrow G$ be a mapping guaranteed by Lemma 3.10 with a structure $S \in \mathcal{S}(G)$ and vertices $\left\{e_{1}, \ldots, e_{n}\right\}$. Then the sets $D_{i}=$ $\left(g^{\prime}\right)^{-1}\left(e_{i}\right)$ for $i \in\{1, \ldots, n\}$ are continua. Since $X$ is locally connected, we can, similarly to the proof of Theorem 2.12(1), slightly enlarge the continua $D_{i}$ to some locally connected, mutually disjoint continua $V_{i}$ containing $D_{i}$. Let $F \in S, \operatorname{dim} F=1$ and $e_{i}, e_{j}$ be the end points of $F$. Then the continuum $D_{F}=\left(g^{\prime}\right)^{-1}(F) \cup V_{i} \cup V_{j}$ is the union of the open subset $\left(g^{\prime}\right)^{-1}\left(F \backslash\left\{e_{i}, e_{j}\right\}\right)$ of $X$ and the locally connected one $V_{i} \cup V_{j}$. So $D_{F}$ is locally connected in view of Lemma 2.10. By Theorem 2.12, including parts (1) and (2), there exists a confluent mapping $g_{F}: D_{F} \rightarrow F$ such that $V_{i} \subset g_{F}^{-1}\left(e_{i}\right), V_{j} \subset g_{F}^{-1}\left(e_{j}\right)$ and the sets $g_{F}^{-1}\left(e_{i}\right), g_{F}^{-1}\left(e_{i}\right)$ are locally connected continua in $X$. Define $g: X \rightarrow G$ as the combination of the mappings $g_{F}$ for all 1-dimensional $F \in S$, i.e. $g(x)=g_{F}(x)$ for $x \in F$, and observe that $g$ is a well defined, continuous, surjective mapping. Note that the set $g^{-1}\left(e_{i}\right)=\bigcup\left\{g_{F}^{-1}\left(e_{i}\right)\right.$ : $\left.F \in S, \operatorname{dim} F=1, e_{i} \in F\right\}$ is a finite union of locally connected continua with nonempty intersection. Thus $g^{-1}\left(e_{i}\right)$ is a locally connected continuum for each $i$. Moreover, for each $F \in S$ with $\operatorname{dim} F=1$ and end points $e_{i}, e_{j}$ the continuum $g^{-1}(F)=D_{F} \cup g^{-1}\left(e_{i}\right) \cup g^{-1}\left(e_{j}\right)$ is a finite union of locally connected continua. Thus $g^{-1}(F)$ is a locally connected continuum. For each $e_{i}$ we have $g^{-1}\left(L_{i}(S)\right) \subset\left(g^{\prime}\right)^{-1}\left(L_{i}(S)\right)$. Therefore the family $\left\{g^{-1}\left(L_{F}(S)\right)\right.$ : $F \in S\}$ refines $\mathcal{U}$. Hence $g$ is as desired.

Corollary 3.12. A continuum $X$ is a locally connected curve if and only if it admits, for every $\varepsilon>0$, a confluent mapping $g: X \rightarrow G$ onto a graph $G$ with a structure $S \in \mathcal{S}(G)$ such that for each $F \in S$ the set $g^{-1}(F)$ is a locally connected continuum of diameter less than $\varepsilon$.

Let $X$ be a compactum, and let $\mathcal{W}$ and $\mathcal{U}=\left(U_{1}, \ldots, U_{n}\right)$ be open covers of $X$. Define $\operatorname{ord}(\mathcal{U})$ as the maximal number of $U_{i}$ 's with nonvoid intersection. Consider the following conditions:

(i) $\operatorname{ord}(\mathcal{U})=2$. 
(ii) $\mathrm{Cl}\left(U_{i}\right) \cap \mathrm{Cl}\left(U_{j}\right) \neq \emptyset$ if and only if $U_{i} \cap U_{j} \neq \emptyset$.

(iii) The cover $\left(\mathrm{Cl}\left(U_{1}\right), \ldots, \mathrm{Cl}\left(U_{n}\right)\right)$ refines the cover $\mathcal{W}$.

(iv) $\operatorname{Bd}\left(U_{i}\right) \cap \operatorname{Bd}\left(U_{j}\right)=\emptyset$ for all $i, j \in\{1, \ldots, n\}$ with $i \neq j$.

It is known [8] that for any 1-dimensional compactum $X$ and any open cover $\mathcal{W}$ of $X$ there exists a cover $\mathcal{U}=\left(U_{1}, \ldots, U_{n}\right)$ that satisfies conditions (i)-(iv). The following lemma is easy to see.

Lemma 3.13. Let $X$ be a compactum and $\mathcal{U}=\left(U_{1}, \ldots, U_{n}\right)$ be an open cover of $X$ that satisfies conditions (i), (ii) and (iv). Then for a sufficiently small positive number $\delta$ and for any $\delta$-mapping $f: X \rightarrow Y$ onto some compactum $Y$ the family $\mathcal{V}=\left(V_{1}, \ldots, V_{n}\right)$, where $V_{i}=\left\{y \in Y: f^{-1}(y) \subset\right.$ $\left.U_{i}\right\}$ for $i \in\{1, \ldots, n\}$, is an open cover of $Y$ that satisfies (i) and (ii).

Let $X$ be a compactum with an open cover $\mathcal{U}=\left(U_{1}, \ldots, U_{n}\right)$ that satisfies conditions (i), (ii), and $G$ be a graph with a structure $S \in \mathcal{S}(G)$ having vertices $e_{1}, \ldots, e_{n}$ such that there is a 1-dimensional element $L_{i, j} \in S$ with end points $e_{i}, e_{j}$ if and only if $U_{i} \cap U_{j} \neq \emptyset$. Let $D_{i, j}=\left(U_{i} \cup U_{j}\right) \backslash \bigcup\left\{U_{k}\right.$ : $j \neq k \neq i, k \in\{1, \ldots, n\}\}$ be defined for all pairs $i, j$ with $U_{i} \cap U_{j} \neq \emptyset$. For every such pair $i, j$, according to the Urysohn lemma, there exists a mapping $g_{i, j}: D_{i, j} \rightarrow L_{i, j}$ such that $g_{i, j}^{-1}\left(e_{i}\right)=U_{i} \backslash \bigcup\left\{U_{k}: k \neq i, k \in\{1, \ldots, n\}\right\}$, $g_{i, j}^{-1}\left(e_{j}\right)=U_{j} \backslash \bigcup\left\{U_{k}: k \neq j, k \in\{1, \ldots, n\}\right\}$. If some set $U_{l}$ does not intersect other elements of $\mathcal{U}$ we let $g_{l}\left(U_{l}\right)=\left\{e_{l}\right\}$. Define $g: X \rightarrow G$ as the combination of all mappings $g_{i, j}$ and $g_{l}$. Then $g$ is a well defined continuous mapping. Observe that $g$ refines $\mathcal{U}$. We call the pair $(G, S)$ the nerve of the cover $\mathcal{U}$, and $g$ a natural map of $X$ into the nerve of $(G, S)$ (compare [8]).

THEOREM 3.14. Every confluently $\mathbb{L} \mathbb{C}$-like compactum $X$ with $\operatorname{dim} X \leq 1$ is confluently graph-like.

Proof. Fix an $\varepsilon>0$ and let $\mathcal{U}=\left(U_{1}, \ldots, U_{n}\right)$ be an open cover of $X$ such that $\operatorname{diam}\left(U_{i}\right)<\varepsilon$ for $i \in\{1, \ldots, n\}$ and the conditions (i), (ii) and (iv) are satisfied. Let $\delta$ be a positive number guaranteed by Lemma 3.13 for the cover $\mathcal{U}$. By the assumption there exists a confluent $\delta$-mapping $f: X \rightarrow Y$ onto a locally connected compactum $Y$. Then the family $\mathcal{V}=\left(V_{1}, \ldots, V_{n}\right)$, where $V_{i}=\left\{y \in Y: f^{-1}(y) \subset U_{i}\right\}$, is an open cover of $Y$ such that (i) and (ii) are satisfied for $\mathcal{V}$. Let $\left(G_{1}, S_{1}\right)$ be the nerve of the cover $\mathcal{V}$ and $g_{1}: Y \rightarrow G_{1}$ be a natural map into the nerve of $\left(G_{1}, S_{1}\right)$. Then $g_{1}$ refines $\mathcal{V}$. By Theorem 3.11 there exists a confluent mapping $g: Y \rightarrow G$ onto a graph $G$ with a structure $S \in \mathcal{S}(G)$ such that the family $\left\{g^{-1}\left(L_{F}(S)\right): F \in S\right\}$ refines $\mathcal{V}$. In particular, $g$ refines $\mathcal{V}$. Define $h=g \circ f: X \rightarrow G$ and note that $h$ is confluent. Since the family $\left\{f^{-1}\left(V_{1}\right), \ldots, f^{-1}\left(V_{n}\right)\right\}$ refines $\mathcal{U}$, we observe that $h$ refines $\mathcal{U}$, i.e. for each $z \in G$ we have $h^{-1}(z) \subset U_{i}$ for some $i \in\{1, \ldots, n\}$. We also have $\operatorname{diam}\left(U_{i}\right)<\varepsilon$. Hence $h: X \rightarrow G$ is a confluent $\varepsilon$-mapping onto a graph $G$. 
The following corollary, a consequence of Theorems 3.2 and 3.14 , is one of the main results of the paper.

Corollary 3.15. For each continuum $X$ the following three conditions are equivalent:

(1) $X$ is confluently graph-representable;

(2) $X$ is confluently graph-like;

(3) $X$ is confluently $\mathbb{L} \mathbb{C}$-like and $\operatorname{dim} X \leq 1$.

COROLLARY 3.16. Each locally connected curve is confluently graph-like, and consequently, it is confluently graph-representable.

We end the paper with the following question that relates this study to the one of homogeneous curves. All known homogeneous curves that contain arcs are confluently graph-like, so the following question naturally appears (compare a discussion of classifying homogeneous curves in [19]).

QUESTION 3.1. Let $X$ be a homogeneous curve that contains an arc. Must $X$ be confluently graph-like?

\section{References}

[1] J. H. Case, Another 1-dimensional homogeneous continuum which contains an arc, Pacific J. Math. 11 (1961), 455-469.

[2] J. J. Charatonik, Confluent mappings and unicoherence of continua, Fund. Math. 56 (1964), 213-220.

[3] J. J. Charatonik, W. J. Charatonik, and J. R. Prajs, Arc property of Kelley and absolute retracts for hereditarily unicoherent continua, Colloq. Math. 97 (2003), to appear.

[4] -, - - - Confluent mappings and the arc property of Kelley, preprint, 2000.

[5] - , - - - Hereditarily unicoherent continua and their absolute retracts, Rocky Mountain J. Math., to appear.

[6] - , - - , Kernels of hereditarily unicoherent continua and absolute retracts, Topology Proc. 26 (2001/02), 127-145.

[7] J. J. Charatonik and J. R. Prajs, On lifting properties of confluent mappings, Proc. Amer. Math. Soc., to appear.

[8] R. Engelking, Dimension Theory, North-Holland and PWN, 1978.

[9] K. R. Gentry, Some properties of the induced map, Fund. Math. 66 (1969), 55-59.

[10] L. H. Hedrick, Properties of inverse systems and mappings between them, Master's thesis, Univ. of Alabama at Birmingham, 1986.

[11] A. Lelek and D. Read, Compositions of confluent mappings and some other classes of functions, Colloq. Math. 29 (1974), 101-112.

[12] S. Mardešić and J. Segal, $\varepsilon$-mappings onto polyhedra, Trans. Amer. Math. Soc. 109 (1963), 146-164.

[13] B. McLean, Confluent images of tree-like curves are tree-like, Duke Math. J. 39 (1972), 465-473.

[14] P. Minc and J. T. Rogers, Jr., Some new examples of homogeneous curves, Topology Proc. 10 (1985), 347-356. 
[15] J. Mioduszewski, Mappings of inverse limits, Colloq. Math. 10 (1963), 39-44.

[16] S. B. Nadler, Jr., Continuum Theory, Dekker, New York, 1992.

[17] E. Puzio, Limit mappings and projections of inverse systems, Fund. Math. 80 (1973), $57-73$.

[18] J. T. Rogers, Jr., Homogeneous continua, Topology Proc. 8 (1983), 213-233.

[19] —, Classifying homogeneous continua, Topology Appl. 44 (1992), 341-352.

[20] A. D. Wallace, Quasi-monotone transformations, Duke Math. J. 7 (1940), 136-145.

[21] G. T. Whyburn, The existence of certain transformations, ibid. 5 (1939), 647-655.

Department of Mathematics

University of Alabama at Birmingham

Birmingham, AL 35294, U.S.A.

E-mail: overstee@math.uab.edu
Institute of Mathematics University of Opole Oleska 48 45-052 Opole, Poland

Current address: Department of Mathematics Idaho State University Pocatello, ID 83209, U.S.A. E-mail: prajs@math.isu.edu

Received 3 June 2002;

in revised form 25 March 2003 